\title{
The combination of PTEN deletion and 16p13.3 gain in prostate cancer provides additional prognostic information in patients treated with radical prostatectomy
}

\author{
Yogesh M. Bramhecha, ${ }^{1,2}$. Shaghayegh Rouzbeh ${ }^{1} \cdot$ Karl-Philippe Guérard $^{1}$ Eleonora Scarlata ${ }^{1}$ Fadi Brimo ${ }^{3}$. \\ Simone Chevalier ${ }^{1,2} \cdot$ Lucie Hamel $^{1} \cdot$ Armen G. Aprikian $^{1} \cdot$ Jacques Lapointe $\mathbb{I}^{1,2}$
}

Received: 27 March 2018 / Revised: 16 June 2018 / Accepted: 16 June 2018 / Published online: 23 August 2018

(c) United States \& Canadian Academy of Pathology 2018

\begin{abstract}
Prostate cancer is a clinically heterogeneous disease and accurately risk-stratifying patients is a key clinical challenge. We hypothesized that the concurrent identification of the DNA copy number alterations 10q23.3 (PTEN) deletion and 16p13.3 (PDPK1) gain, related to the PI3K/AKT survival pathway, would improve prognostication. We assessed PTEN deletion status using fluorescence in situ hybridization (FISH) and evaluated its clinical significance in combination with the 16p13.3 gain in a set of 332 primary radical prostatectomy cases on a tissue microarray with clinical follow-up. The PTEN deletion was detected in 34\% (97/287) of the evaluable tumors and was significantly associated with high Gleason grade group $(P<$ $0.0001)$ and advanced pathological tumor stage (pT-stage, $P<0.001$ ). The $P T E N$ deletion emerged as a significant predictor of biochemical recurrence independent of the standard clinicopathologic parameters (hazard ratio: $3.00,95 \%$ confidence interval: $1.81-4.98 ; P<0.0001)$ and further stratified patients with low and intermediate risk of biochemical recurrence [Gleason grade group $1-2(\leq 3+4)$, Gleason grade group $2(3+4)$, pT2, prostate-specific antigen $\leq 10$, low and intermediate CAPRA-S score; $\log$-rank $P \leq 0.007]$. A $P T E N$ deletion also increased the risk of distant metastasis (log-rank, $P=0.001)$, further supporting its role in prostate cancer progression. Combining both $16 \mathrm{p} 13.3$ gain and PTEN deletion improved biochemical recurrence risk stratification and provided prognostic information beyond the established CAPRA-S score (coalteration: hazard ratio: $4.70,95 \%$ confidence interval: $2.12-10.42 ; P<0.0001)$. Our study demonstrates the potential clinical utility of PTEN genomic deletion in low-intermediate risk patients and highlights the enhanced prognostication achieved when assessed in combination with another genomic biomarker related to the PI3K/AKT pathway, thereby supporting their promising usefulness in clinical management of prostate cancer.
\end{abstract}

\section{Introduction}

Prostate cancer remains a major clinical burden, being the most prevalent cancer and one of the leading causes of

Jacques Lapointe

jacques.lapointe@mcgill.ca

1 Division of Urology, Department of Surgery, McGill University and the Research Institute of the McGill University Health Centre, Montreal, QC, Canada

2 Division of Experimental Medicine, McGill University and the Research Institute of the McGill University Health Centre, Montreal, QC, Canada

3 Department of Pathology, McGill University and the Research Institute of the McGill University Health Centre, Montreal, QC, Canada cancer-specific deaths in North American men [1]. It is a clinically heterogeneous disease wherein the majority of cancers display a favorable outcome, while a subset affecting a considerable number of patients progress to metastatic and lethal stage [2]. Radical prostatectomy or radiation therapy is considered the standard primary treatment option for localized prostate cancer and more recently, active surveillance has emerged as a viable alternative for patients presenting favorable clinicopathologic features [3]. One of the key challenges in the clinical management of prostate cancer is to accurately distinguish indolent from aggressive tumors in order to avoid overtreatment of clinically insignificant cancers and undertreatment of tumors with metastatic potential [4].

Serum prostate-specific antigen levels, biopsy Gleason grade and clinical tumor stage (cT-stage) are used to risk stratify patients, but are not sufficient to accurately predict 
individual clinical outcome. Assessing the Gleason grade based on prostate biopsies is challenging and frequently leads to an underestimation of the actual grade of the entire tumor burden [5]. To address the shortcomings of the clinicopathologic predictors and to better capture the clinical heterogeneity of prostate cancer, biomarkers are being developed based on the underlying spectrum of molecular abnormalities of this disease. DNA copy number alterations are common in cancer and have been associated with molecular subtypes of prostate cancer, supporting the existence of alternative parallel pathways of tumorigenesis $[6,7]$. Copy number alterations such as deletion of chromosome 10q23 (PTEN) and gain of $16 \mathrm{p} 13.3(P D P K 1)$ are detected at higher frequency in lymph node metastases than in primary tumors, suggesting a role in prostate cancer progression [7, 8]. The phosphatase and tensin homolog deleted on chromosome 10 (PTEN) protein is a tumor suppressor that negatively regulates the phosphatidylinositol 3-kinase/AKT survival pathway [9-11]. Encoded by PDPK1, 3Phosphoinositide-dependent protein kinase-1 (PDK1) phosphorylates and activates the AGC kinase members regulated by phosphatidylinositol 3-kinase, including AKT [12]. In addition to its kinase activity on AKT, our laboratory has shown that PDK1 also has an important role in prostate cancer cell migration [13]. PTEN deletion has been shown to be associated with poor outcome in prostate cancer after radical prostatectomy [13-17]. Recently, we reported that the 16 p13.3 gain was also associated with adverse outcome after radical prostatectomy [18]. In the current study, we demonstrated that the PTEN genomic deletion measured by FISH is a strong independent predictor of poor clinical outcome after radical prostatectomy, including in low-intermediate risk patients and showed that the combination of PTEN deletion and 16p13.3 gain status improved patient risk stratification.

\section{Materials and methods}

\section{Study population and tissue microarray}

This study was done in compliance with the REMARK guidelines [19] and approved by the Research Ethics Board of the McGill University Health Centre (BDM-10-115) with the written informed consent of the participants. A set of 332 de-identified formalin-fixed paraffin-embedded (FFPE) radical prostatectomy specimens collected between 1993 and 2008 at the McGill University Health Centre were represented on a tissue microarray by duplicate $1 \mathrm{~mm}$ cores extracted from the dominant tumor nodule. Dominant nodule was defined as generally the largest nodule. In cases
Table 1 Clinicopathologic features of radical prostatectomy cases represented on the tissue microarray

\begin{tabular}{|c|c|c|}
\hline $\begin{array}{l}\text { Clinicopathologic } \\
\text { variables }\end{array}$ & Category & $n(\%)$ \\
\hline Total number of cases & $n$ & 332 \\
\hline Age (years) & $\begin{array}{l}\text { Median } \\
\text { Min-max }\end{array}$ & $\begin{array}{l}61 \\
43-73\end{array}$ \\
\hline $\begin{array}{l}\text { Preoperative serum } \\
\text { prostate-specific antigen } \\
(\mathrm{ng} / \mathrm{ml})\end{array}$ & $\begin{array}{l}n^{\mathrm{a}} \\
\text { Mean }( \pm \mathrm{SD}) \\
\text { PSA } \leq 10 \\
\text { PSA }>10\end{array}$ & $\begin{array}{l}327 \\
8.66( \pm 8.27) \\
253(77 \%) \\
74(23 \%)\end{array}$ \\
\hline $\begin{array}{l}\text { Gleason grade groups at } \\
\text { surgery }\end{array}$ & $\begin{array}{l}\text { Group } 1(3+3) \\
\text { Group } 2(3+4) \\
\text { Group } 3(4+3) \\
\text { Group } 4(8) \\
\text { Group } 5(\geq 9)\end{array}$ & $\begin{array}{l}70(21 \%) \\
153(46 \%) \\
78(24 \%) \\
11(3 \%) \\
20(6 \%)\end{array}$ \\
\hline $\begin{array}{l}\text { Pathological stage } \\
\text { (T-stage) }\end{array}$ & $\begin{array}{l}\text { pT2 } \\
\text { pT3a } \\
\text { pT3b }\end{array}$ & $\begin{array}{l}219(66 \%) \\
91(27 \%) \\
22(7 \%)\end{array}$ \\
\hline Surgical margin status & Positive & $97(29 \%)$ \\
\hline Follow-up (months) & $\begin{array}{l}n^{\mathrm{a}} \\
\text { Median (min-max) }\end{array}$ & $\begin{array}{l}297 \\
116(1-253)\end{array}$ \\
\hline Biochemical recurrence & $\begin{array}{l}n^{\mathrm{a}} \\
\text { Positive }\end{array}$ & $\begin{array}{l}297 \\
81(27 \%)\end{array}$ \\
\hline Distant metastases & Positive & $16 / 321^{\mathrm{a}}(5 \%)$ \\
\hline
\end{tabular}

${ }^{\mathrm{a}}$ Values not available for all the 332 cases ( $n$ noted for each variable)

in which a smaller nodule was considered to be prognostically more significant (higher grade or stage), this smaller nodule was considered to be dominant.

The clinical information was retrieved from the medical charts and the pathological correlates were obtained after rereview of all the radical prostatectomy cases by a single dedicated genitourinary pathologist (F.B.). The final Gleason grade was assigned according to the latest International Society of Urological Pathology/World Health Organization recommendations [20]. The clinicopathologic characteristics of 303 of the 332 cases were reported previously [18] and those of the entire expanded cohort are summarized in Table 1. The mean preoperative serum prostate-specific antigen level was $8.66( \pm 8.27)$ and the distribution of Gleason grade group 1 (Gleason score 6), 2 (Gleason score $3+4$ ), 3 (Gleason score $4+3$ ), 4 (Gleason score 8), and 5 (Gleason score $\geq 9$ ) was $21 \%, 46 \%, 24 \%, 3 \%$, and $6 \%$, respectively. Sixty-six percent of patients were at stage pT2 while $34 \%$ belonged to stage pT3. Patients receiving neoadjuvant hormone therapy $(n=6)$ and cases with missing serum prostatespecific antigen data post-radical prostatectomy $(n=15)$ were not included in the biochemical recurrence analyses. Surgical failure cases $(n=14)$, for which the serum prostate-specific antigen did not fall to undetectable levels post-radical prostatectomy, were also excluded from the biochemical recurrence analyses. No patient had received adjuvant radiation therapy after surgery. The primary endpoint of the study was 
biochemical recurrence and was defined by a serum prostatespecific antigen elevation of $>0.2 \mathrm{ng} / \mathrm{ml}$ following radical prostatectomy $(27 \%)$. The recurrence-free interval was defined as the time between the surgery date and the date of the first prostate-specific antigen increase above $0.2 \mathrm{ng} / \mathrm{ml}$. Patients without biochemical recurrence event were censored at the last follow-up date with prostate-specific antigen measurement. The median follow-up for the cohort was 116 months (1-253 months, min-max). Metastasis status was evaluated and confirmed by imaging in patients with clinical symptoms $(n=16)$. The metastasis-free interval was defined as the period between the surgery date and the date of first metastasis detection and patients without signs/symptoms related to metastasis were censored at the last follow-up/ prostate-specific antigen date. The CAPRA-S (Cancer of the Prostate Risk Assessment Post-Surgical) score was calculated from the status of six clinicopathologic variables [preoperative prostate-specific antigen, Gleason score, surgical margins, extracapsular extension, seminal vesicle invasion, lymph node invasion], and each patient was assigned to one of the three risk groups: low (0-2), intermediate [3-5], and high ( $\geq 6)$ according to Cooperberg et al. [21]. Of note, patients who did not undergo a lymph node dissection were considered to have negative lymph node for CAPRA-S score calculation as previously described [22]. The chromosome 16p13.3 gain data recently reported by our lab for this cohort [18] was used for the combinatorial approach.

\section{Fluorescence in situ hybridization (FISH)}

The BAC clone CTD-2557P6 (BACPAC Resources Center, Oakland, CA) mapping to the PTEN gene on the chromosome 10q23.3 region and commercially available CEP10 Spectrum Green probe (CEP 10, Abbott Molecular, Abbott Park, IL), which spans the 10p11.1-q11.1 centromeric region were used to perform dual-color FISH on the $5 \mu \mathrm{m}$ tissue microarray sections as we described previously [13]. The CTD-2557P6 DNA was labeled with the Spectrum Orange-dUTP (Enzo Life Science, Farmingdale, NY) using the Nick Translation Reagent Kit (Abbott Molecular) as per the kit manual.

\section{FISH data analysis}

To evaluate the PTEN copy number status, fluorescent signals were counted in 100 non-overlapping interphase nuclei for each case (as identified on corresponding H\&E) counterstained with ProLong ${ }^{\circledR}$ Gold antifade reagent with DAPI (Life Technology, CA), to delineate nuclei. The PTEN deletion was defined as $\geq 15 \%$ of tumor nuclei containing one or no PTEN locus signal and by the presence of two CEP10 signals as we previously reported [13]. A tumor was considered homozygous-deleted if $\geq 15 \%$ of tumor nuclei had no PTEN locus signals and two CEP10 signals. Images were acquired with an Olympus IX-81 inverted microscope at $\times 96$ magnification, using Image-Pro Plus 7.0 software (Media Cybernetics, Rockville, MD).

\section{Statistical analysis}

The association between copy number alterations and the clinicopathologic indicators were assessed by Fisher's exact test for categorical variables and unpaired $t$-test for continuous variables. Kaplan-Meier curves were generated for biochemical recurrence-free and metastasis-free survival analysis. The log-rank test was used to evaluate the significance of differences between the stratified survival functions. Cox regression analyses were used to evaluate univariate hazard ratios and multivariate Cox proportional hazards regression analysis was performed to identify independent predictors of biochemical recurrence. The $\mathrm{C}$-index was calculated as described by Harrel et al. [23]. Analyses were performed using SPSS, WinStat, and R (Version 3.3.2).

\section{Results}

\section{Association of PTEN deletion status with adverse clinical outcome post-radical prostatectomy}

The 10q23.3 (PTEN) deletion status was assessed using dual-color FISH on 332 radical prostatectomy specimens represented on a tissue microarray. The clinicopathologic features of these patients are summarized in Table 1. The PTEN deletion status could be successfully assessed in 287 tumors arrayed out, of which 97 (34\%) harbored a PTEN genomic deletion. The PTEN deletion status was consistent across duplicate TMA cores evaluated. Of the cases with PTEN deletion, 80 (28\%) were hemizygous deleted while 17 (6\%) harbored a homozygous PTEN deletion (Fig. 1). Of note, 15 out the 17 cases that we identified as homozygousdeleted for PTEN also harbored a significant number of nuclei $(\geq 15 \%)$ showing a hemizygous deletion within the tissue microarray core. Preliminary analysis indicated that these cases with homozygous deletion were not different than cases harboring only a hemizygous deletion in term of their association with adverse pathology and poor outcome (not shown). We therefore considered hemizygous and homozygous PTEN deletion cases together as a single group for the analyses presented in this report. As shown in Table 2, the PTEN deletion status was significantly associated with high Gleason grade group $(P=0.0001)$ and advanced pT-stage $(P=0.001)$.

The prognostic significance of PTEN genomic status was first evaluated using biochemical recurrence as a surrogate primary endpoint post-radical prostatectomy. PTEN FISH 

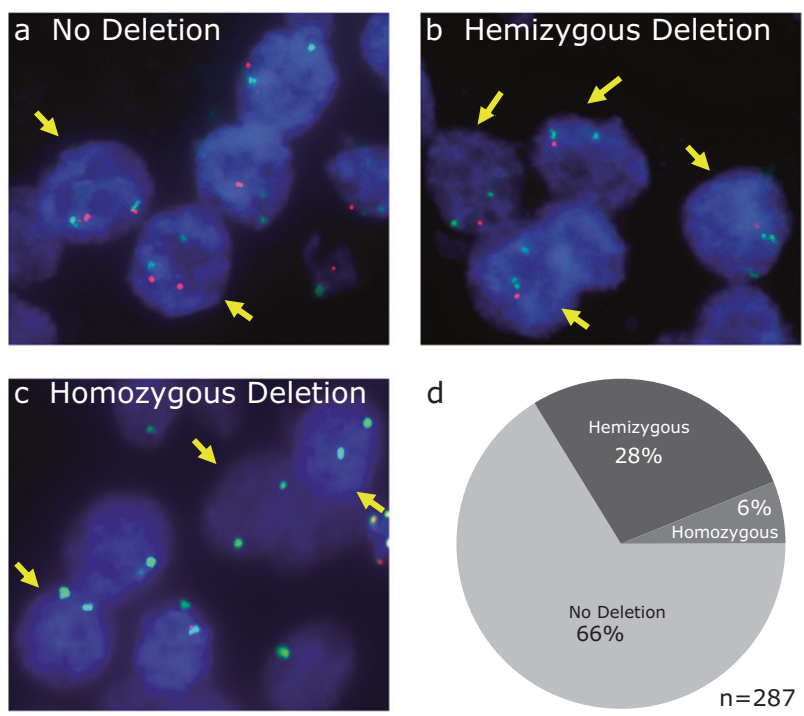

Fig. 1 Dual-color FISH analysis of PTEN (10q23) deletion in prostate cancer specimens. White arrows show (a) normal interphase nuclei with 2 green and 2 orange signals in a PCa tumor with no PTEN deletion; (b) 2 green and 1 orange signals in a tumor harboring hemizygous PTEN deletion; and (c) 2 green and 0 orange signals in a homozygous PTEN-deleted case. FISH analysis (d) detected hemizygous in 80/287 (28\%), homozygous in 17/287 (6\%), and no PTEN deletion in $189 / 287(66 \%)$ of the primary radical prostatectomy samples on the McGill urology tissue microarray $(n=287)$

Table 2 Association of PTEN deletion status with clinicopathologic features of aggressive prostate cancer

\begin{tabular}{|c|c|c|c|c|}
\hline \multirow{2}{*}{$\begin{array}{l}\text { Clinicopathologic } \\
\text { variables }\end{array}$} & \multirow{2}{*}{$\begin{array}{l}\text { Total } \\
\text { cases } \\
n(\%)\end{array}$} & \multicolumn{2}{|l|}{ PTEN status } & \multirow[t]{2}{*}{$P$-value } \\
\hline & & No deletion & Deletion & \\
\hline PTEN status & 287 & $190(66 \%)$ & $97(34 \%)$ & \\
\hline Gleason grade groups & 287 & & & 0.0001 \\
\hline Group 1 (GS $3+3)$ & 61 & $52(85 \%)$ & $9(15 \%)$ & \\
\hline Group 2 (GS $3+4)$ & 132 & $90(68 \%)$ & $42(32 \%)$ & \\
\hline Group 3 (GS $4+3)$ & 67 & $37(55 \%)$ & $30(45 \%)$ & \\
\hline Group 4 and $5(\mathrm{GS} \geq 8)$ & 27 & $11(41 \%)$ & $16(59 \%)$ & \\
\hline Pathological T-stage & 287 & & & 0.001 \\
\hline pT2 & 189 & $138(73 \%)$ & $51(27 \%)$ & \\
\hline pT3a & 77 & $43(56 \%)$ & $34(44 \%)$ & \\
\hline pT3b & 21 & $9(43 \%)$ & $12(57 \%)$ & \\
\hline $\begin{array}{l}\text { Preoperative prostate- } \\
\text { specific antigen (mean/ } \\
\pm \text { standard deviation) }\end{array}$ & 282 & $7.95( \pm 7.60)$ & $9.03( \pm 7.24)$ & $0.25^{\mathrm{a}}$ \\
\hline Surgical margin status & 287 & & & 0.30 \\
\hline Negative & 200 & $136(68 \%)$ & $64(32 \%)$ & \\
\hline Positive & 87 & $54(62 \%)$ & $33(38 \%)$ & \\
\hline
\end{tabular}

$P$-value calculated by Fisher exact test

Number of cases that could be assessed $(n)$ noted for each variable ${ }^{\mathrm{a}}$ Unpaired $t$-test status and complete prostate-specific antigen follow-up data were available for 256 radical prostatectomy cases, out of which 69 (27\%) experienced biochemical recurrence. The PTEN genomic deletion status emerged as a significant predictor of early biochemical recurrence following radical prostatectomy (log-rank $P<0.0001$; Fig. 2a) independent of the standard clinicopathologic prognostic indicators like Gleason grade group, pT-stage, preoperative prostatespecific antigen level, and surgical margin status in a multivariate Cox analysis (hazard ratio: 3.00, 95\% confidence interval: 1.81-4.99; $P<0.0001$; Table 3A).

\section{Clinical significance of PTEN genomic deletion in low-intermediate risk prostate cancer patients}

We assessed the ability of the PTEN deletion status to predict biochemical recurrence risk in a clinically relevant subset of patients belonging to grade groups 1 and $2(\leq 3+$ 4) according to the latest International Society of Urological Pathology/World Health Organization Gleason grading recommendations [20]. The PTEN deletion status was significantly associated with biochemical recurrence in patients of grade group 1-2 $(\leq 3+4$, log-rank, $P<0.0001$, Fig. 2 b) including those that were also of stage pT2 and with preoperative prostate-specific antigen $\leq 10$ (log-rank, $P=$ 0.002 , Fig. 2c). Furthermore, the PTEN deletion was significantly linked to biochemical recurrence in a subgroup of grade group $2(3+4$, log-rank, $P<0.0001$, Fig. 2 d) even with favorable stage pT2 and prostate-specific antigen $\leq 10$ (log-rank, $P=0.007$, Fig. 2e). There was an insufficient number of biochemical recurrence events $(n=1)$ in grade group 1 (Gleason score 6) to allow subgroup analysis and the PTEN deletion status did not further stratify grade group 3-5 ( $\geq 4+3$, not shown). We then assessed if the PTEN genomic deletion status could further stratify the risk groups defined by the clinically validated clinicopathologic CAPRA-S score to predict biochemical recurrence postradical prostatectomy [21]. The multivariate analysis showed that the PTEN deletion was a significant predictor of biochemical recurrence along CAPRA-S score risk groups (hazard ratio: 2.84 , 95\% confidence interval: 1.75 4.63; $P<0.0001$, Table 3B). PTEN deletion identified a subset of patients with a greater risk of biochemical recurrence among those of low and intermediate CAPRA-S score risk groups (Fig. 2f-g, log-rank, $P=0.0001$ and $P=$ 0.0002 , respectively). We further evaluated the association of PTEN deletion with bone or soft tissue metastases, an important adverse secondary endpoint. PTEN deletion status was indeed significantly associated with an increased risk of distant metastasis (log-rank $P=0.001$, Fig. 2h), further supporting its potential clinical utility as a marker of prostate cancer progression. 


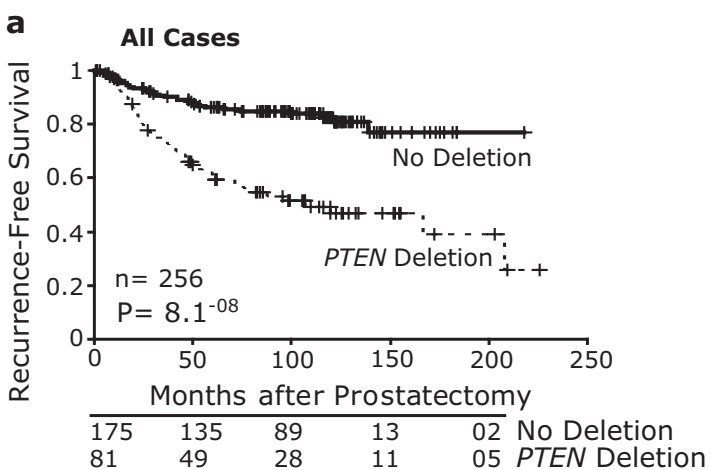

C

Cases with Grade Group 1-2 $(\leq 3+4)$, pT2

\& Prostate Specific Antigen $\leq 10$
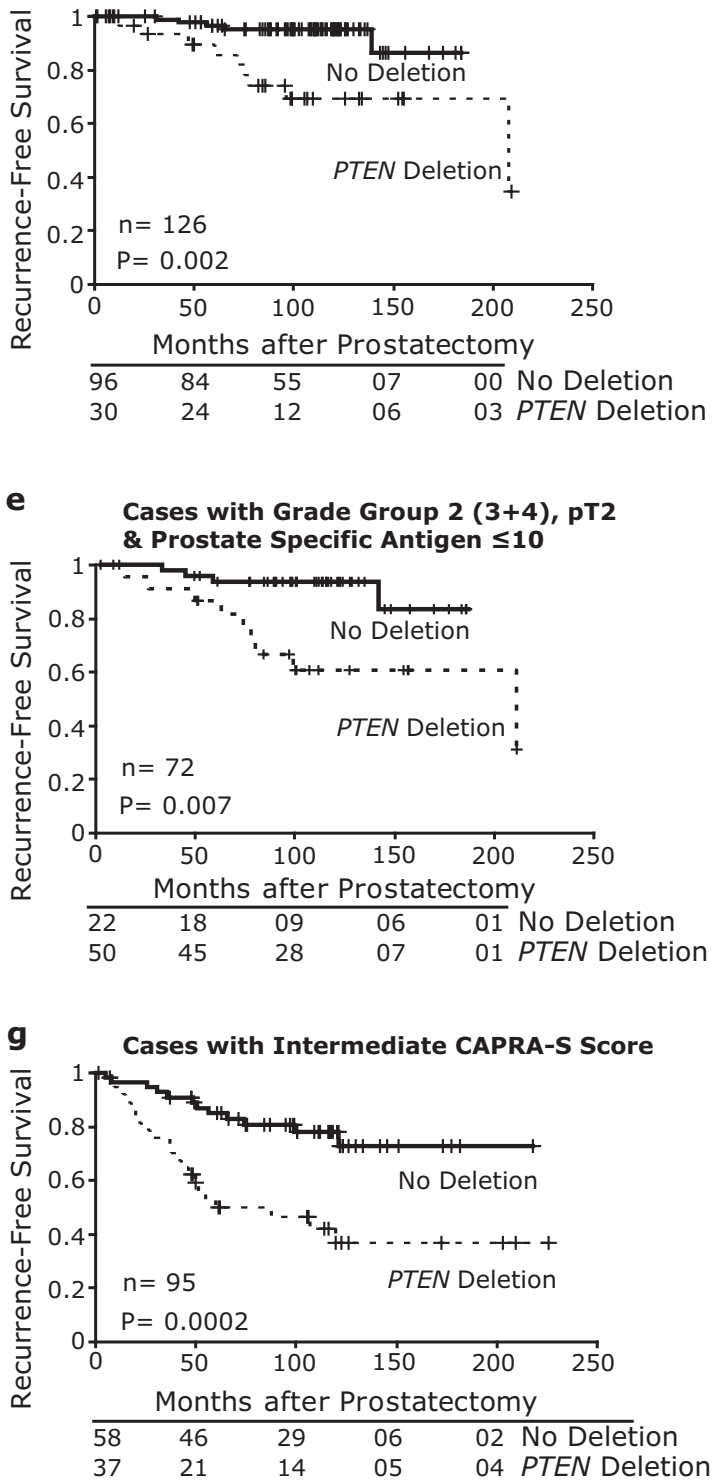

b

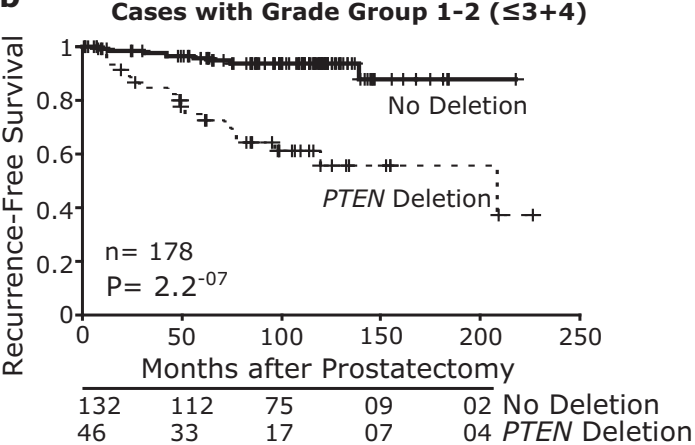

d

Cases with Grade Group $2(3+4)$

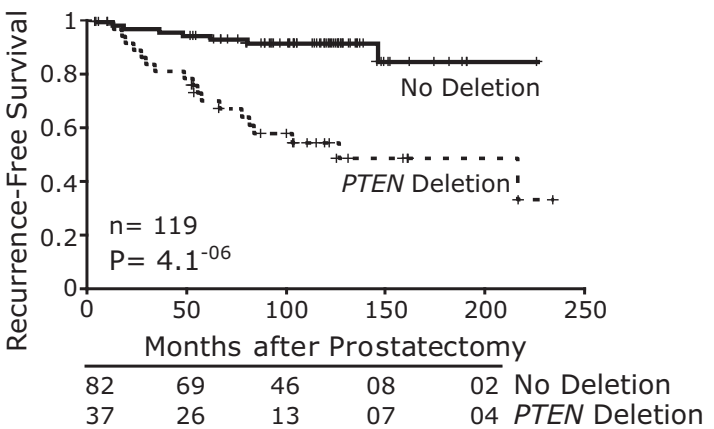

Cases with Low CAPRA-S Score
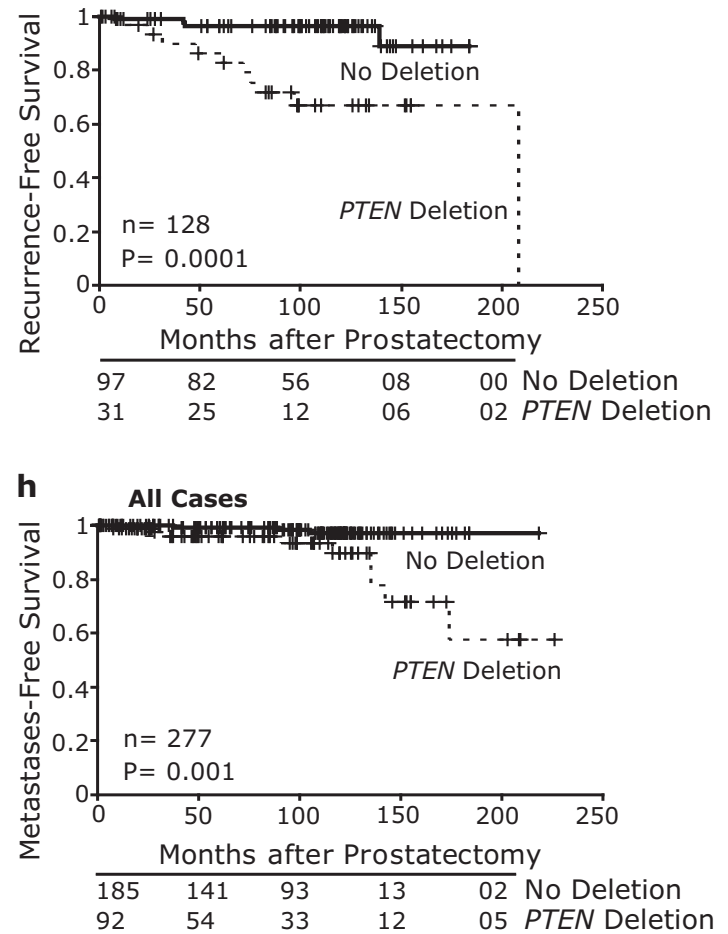
Fig. 2 Prognostic value of the PTEN genomic deletion in prostate tumors. Kaplan-Meier recurrence-free survival analysis of patients stratified on the basis of PTEN deletion status determined by FISH in (a) all radical prostatectomy patients with clinical follow-up for biochemical recurrence; (b) Gleason grade group 1-2 $(\leq 3+4)$, (c) Gleason grade group 1-2 $(\leq 3+4)$, stage pT2 and prostate-specific antigen $\leq 10$ patients; (d) Gleason grade group $2(3+4)$; (e) Gleason grade group $2(3+4)$, stage pT2 and prostate-specific antigen $\leq 10$ patients; (f) low CAPRA-S score (0-2) risk patients; and (g) intermediate CAPRA-S score [3-5] risk patients. Survival analysis based on PTEN deletion status shows (h) worse metastases-free survival. Censored data (tick marks), number of patients at risk at respective time points, and $P$-value (log-rank test) are indicated

\section{Improved biochemical recurrence risk stratification upon combining 16p13.3 gain with PTEN deletion}

16 p13.3 genomic gain status has recently been shown to be associated with aggressive clinicopathologic features of prostate cancer $[8,18]$, as well as with poor clinical outcome in our cohort [18]. A set of 251 cases for which both 16 p13.3 gain and PTEN deletion data were available was used for the combinatorial PTEN-16p13.3 co-alteration analyses. We first tested whether PTEN deletion status could further stratify patients without $16 \mathrm{p} 13.3$ gain. As shown in Fig. 3a, cases with PTEN deletion have an increased risk of biochemical recurrence among this subgroup (log-rank, $P<0.0001)$. Interestingly, amongst patients with no PTEN deletion, the $16 \mathrm{p} 13.3$ gain further identified a subset of patients at high risk of recurrence (Fig. 3b, log-rank, $P=0.001$ ). We then grouped cases based on their PTEN-16p13.3 co-alteration status-(0) no PTEN deletion and no 16p13.3 gain, (1) PTEN deletion or 16p13.3 gain, and (2) PTEN deletion and 16p13.3 gain. Kaplan-Meier analysis demonstrated that the PTEN16 p13.3 co-alteration status further segregated prostate cancer cases in three distinct prognostic subgroups (logrank $P<0.0001$, Fig. 3c) stratified by the number of positive markers: the favorable prognostic group with no alterations in PTEN and 16p13.3, the intermediate prognostic group with one alteration in either PTEN or 16p13.3, and the worst prognostic group with two alterations (PTEN and 16p13.3). Moreover, in the multivariate Cox analysis adjusted for standard prognostic indicators, the PTEN16 p13.3 co-alteration status remained significant and conferred the highest risk of biochemical recurrence (hazard ratio: $4.18,95 \%$ confidence interval: $1.82-9.59 ; P=0.001$; Table 4A). Similarly, a PTEN deletion along a 16p13.3 gain increased the risk of recurrence significantly even after adjusting for the CAPRA-S score risk groups (hazard ratio: 4.70, 95\% confidence interval: $2.12-10.42, P<0.0001$, Table 4B). To estimate the potential prognostic benefit of assessing both genomic alterations, we calculated the Cindex for each of PTEN deletion and 16p13.3 gain alone and in combination using biochemical recurrence as an endpoint in Cox model. The C-index was higher by using both alterations than $16 \mathrm{p} 13.3$ gain or PTEN deletion alone (0.69 vs. 0.62 and 0.63 , respectively) and each of these alterations improved the C-index of the CAPRA-S score reaching a maximum when both were included $(0.78$, Fig. 3d).

\section{Discussion}

In this study, we confirmed the association of PTEN deletion with poor outcome in prostate cancer and demonstrated its potential at further stratifying low-intermediate risk patients treated by radical prostatectomy. In addition, we showed that its prognostic value can be improved by considering the gain of $16 \mathrm{p} 13.3$. We detected PTEN deletion by FISH in $34 \%$ of the 287 radical prostatectomy specimens examined, a frequency falling within the range of $17-42 \%$ reported by other previously published studies using FISH and including over hundred samples [14-17, 24, 25]. The majority of deletions observed were hemizygous ( $28 \%$ vs. $6 \%$ of homozygous) in agreement with most of the previous publications on radical prostatectomy cases. In contrast, Krohn et al. [14] reported $12 \%$ of homozygous and $8 \%$ of hemizygous deletion in their cohort while Troyer et al. [16] observed 9\% homozygous and 9\% hemizygous deletion in their samples. The variation in frequency of PTEN deletion and in proportion of hemizygous vs. homozygous deletion observed among the studies possibly reflects differences of cohort sizes and clinicopathologic features, but also likely differences in tissue preparation and FISH scoring method. The presence of homozygous-deleted and hemizygousdeleted nuclei in most of tumor classified as PTEN homozygous-deleted in our study likely reflect intratumoral heterogeneity and possibly disease progression.

Supporting a role for PTEN alteration in prostate cancer progression, our study showed that its deletion was significantly associated with the aggressive clinicopathologic features of high Gleason grade group and advanced surgical stage pT3, a finding consistent with previous reports of PTEN FISH on large radical prostatectomy sets $[14,16]$. In agreement with our prior report on a separate sample set [13] as well as with previous studies of other groups [1417], we have also shown that PTEN deletion assessed by FISH was associated with biochemical recurrence after radical prostatectomy. Moreover, the prognostic value of the deletion was independent of standard clinicopathologic markers. In our study, homozygous deletion was not associated with a higher risk of biochemical recurrence than hemizygous deletion in agreement with the report of Krohn et al. [14], but in contrast to Yoshimoto et al. and Troyer et al. $[16,17]$. Our data indicate that the loss of one copy was sufficient to increase significantly the risk of 
Table 3 Univariate and multivariate Cox proportional hazard analysis predicting biochemical recurrence for PTEN deletion status adjusted for standard clinicopathologic parameters (A) and CAPRA-S score (B)

\begin{tabular}{|c|c|c|c|c|}
\hline \multirow[t]{2}{*}{ Variables } & \multicolumn{2}{|l|}{ Univariate analysis } & \multicolumn{2}{|l|}{ Multivariate analysis } \\
\hline & $\begin{array}{l}\text { Hazard ratio ( } 95 \% \\
\text { confidence interval) }\end{array}$ & $P$-value & $\begin{array}{l}\text { Hazard ratio ( } 95 \% \\
\text { confidence interval) }\end{array}$ & $P$-value \\
\hline \multicolumn{5}{|c|}{ (A) Standard clinicopathologic parameters } \\
\hline $\begin{array}{l}P T E N \text { status (deleted vs. } \\
\text { non-deleted) }\end{array}$ & $3.47(2.14-5.63)$ & $<0.0001$ & $3.00(1.81-4.99)$ & $<0.0001$ \\
\hline $\begin{array}{l}\text { Preoperative prostate-specific } \\
\text { antigen }^{\mathrm{a}}\end{array}$ & $1.06(1.04-1.09)$ & $<0.0001$ & $1.05(1.01-1.08)$ & 0.005 \\
\hline \multicolumn{5}{|l|}{ Gleason grade } \\
\hline $\begin{array}{l}\text { Group } 3-5(\geq 4+3) \text { vs. } \\
\text { group } 1-2(\leq 3+4)\end{array}$ & $4.75(2.91-7.78)$ & $<0.0001$ & $2.60(1.44-4.67)$ & 0.001 \\
\hline pT-stage (T3 vs. T2) & $3.32(2.05-5.38)$ & $<0.0001$ & $1.53(0.87-2.67)$ & 0.137 \\
\hline $\begin{array}{l}\text { Surgical margin (positive vs. } \\
\text { negative) }\end{array}$ & $2.30(1.43-3.72)$ & 0.001 & $1.88(1.12-3.13)$ & 0.016 \\
\hline Age at surgery ${ }^{\mathrm{a}}$ & $1.02(0.98-1.07)$ & 0.256 & $0.98(0.94-1.03)$ & 0.494 \\
\hline \multicolumn{5}{|l|}{ (B) CAPRA-S score } \\
\hline $\begin{array}{l}P T E N \text { status (deleted vs. } \\
\text { non-deleted) }\end{array}$ & $3.47(2.14-5.63)$ & $<0.0001$ & $2.84(1.75-4.63)$ & $<0.0001$ \\
\hline \multicolumn{5}{|l|}{ CAPRA-S risk } \\
\hline Low $(0-2)$ reference & - & $<0.0001$ & - & $<0.0001$ \\
\hline Intermediate $(3-5)$ & $3.40(1.81-6.36)$ & $<0.0001$ & $3.00(1.60-5.64)$ & 0.001 \\
\hline High $(\geq 6)$ & $10.65(5.42-20.91)$ & $<0.0001$ & $8.95(4.53-17.67)$ & $<0.0001$ \\
\hline
\end{tabular}

${ }^{a}$ Analyzed as a continuous variable; $P$-value: Wald test biochemical recurrence, which is consistent with PTEN haploinsufficiency demonstrated in prostate cancer animal models [26]. It is also possible that the second allele has been inactivated by alternative mechanisms [27], which was not investigated in our study. Interestingly, the PTEN deletion status could further stratify patients of lowintermediate risk grade group 1-2 $(\leq 3+4)$, pT2, and prostate-specific antigen $<10$, a finding not reported in previous studies. The revised Gleason scoring system applied to our cohort offers more refinement over previous iterations by splitting Gleason score 7 into two groups of distinct prognoses: grade group $2(3+4)$ and grade group 3 $(4+3)$. While the grade group 2 has the best outcome, it was further stratified by PTEN FISH. Since the percentage of Gleason pattern 4 was not recorded in this cohort, it is unclear if and how it would correlate with the PTEN status. Similarly, PTEN FISH was able to sub-classify cases belonging to low and intermediate CAPRA-S risk groups, thus emphasizing the potential complementary role of this marker to clinicopathologic assessment for outcome prediction.

PTEN deletions detected by FISH are known to be enriched in metastatic as compared to primary prostate tumors [25, 28]. To our knowledge, one PTEN FISH study reported metastasis outcome on radical prostatectomy specimens, but did not find any significant association with PTEN deletion [16]. Here, we have shown that patients with a PTEN deletion in their radical prostatectomy sample were at a higher risk of experiencing distant metastases. Our results are in agreement with Lotan et al. [29] who used a selected high-risk radical prostatectomy cohort (all patients experienced a biochemical recurrence) to demonstrate that the loss of PTEN protein expression was associated with a shorter time to distant metastasis. While further validation on different cohorts is needed, our findings highlight the potential of PTEN deletion as a marker of disease progression to advanced metastatic disease.

We recently reported that the $16 \mathrm{p} 13.3$ gain was associated with aggressive clinicopathologic features of prostate cancer as well as an increased risk of biochemical recurrence and distant metastases in the same radical prostatectomy specimens surveyed here for PTEN deletion [18]. Moreover, the $16 \mathrm{p} 13.3$ gain status improved the stratification of patients with intermediate and high risk of disease progression based on their CAPRA-S score. The analysis of the combined data presented here exemplified the advantages of considering both PTEN and 16p13.3 CNAs for biochemical recurrence risk stratification. Cases that were negative for PTEN deletion were further stratified by the $16 p 13.3$ gain status and vice versa, thus allowing the identification of patients that have a reduced risk of biochemical recurrence. A maximum risk of biochemical recurrence was reached for patients whose tumors harbored both PTEN deletion and 16p13.3 gain. The advantage of this combinatorial approach was further evidenced by an increase of the $\mathrm{C}$-index, which reached its maximum when 


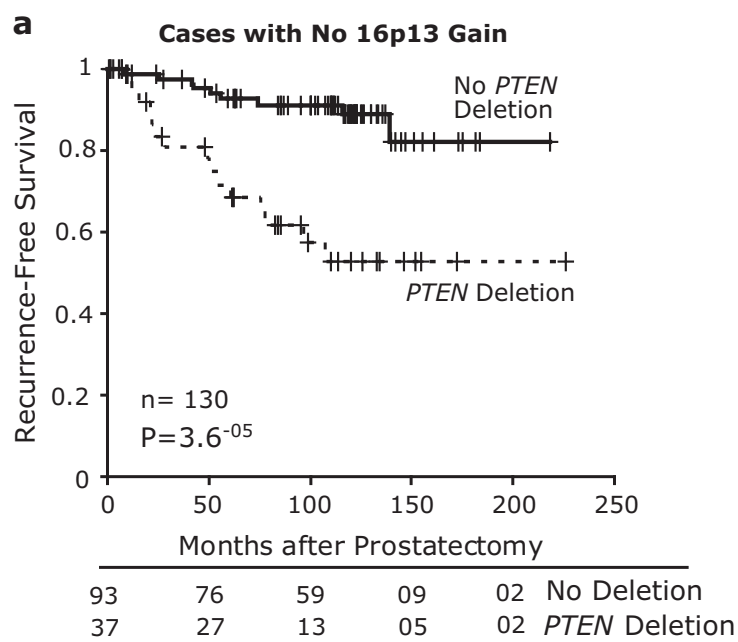

C All Cases Stratified by PTEN \& 16 p13 Status

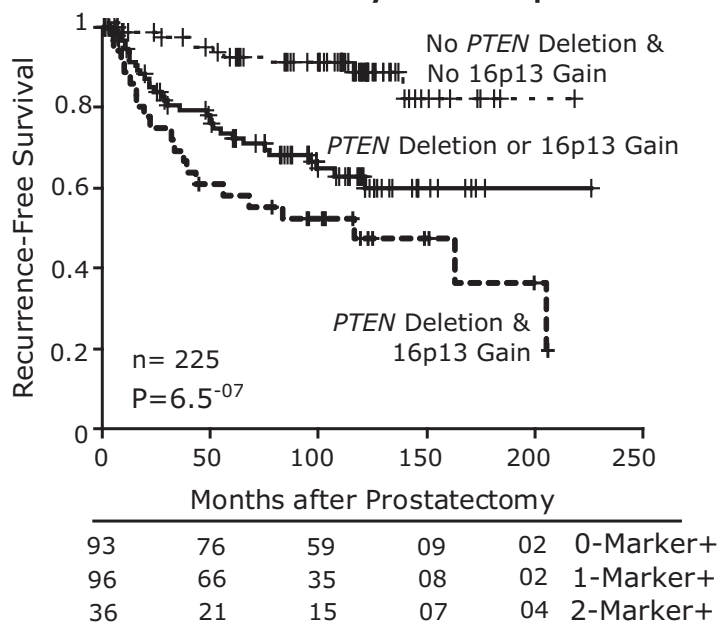

Fig. 3 Improved risk stratification upon combination of $16 \mathrm{p} 13.3$ gain and PTEN genomic deletion. Kaplan-Meier recurrence-free survival analysis of prostate cancer patients stratified on the basis of (a) PTEN deletion status in cases harboring no $16 \mathrm{p} 13$ gain; (b) $16 \mathrm{p} 13$ gain status in cases harboring no PTEN deletion; and (c) in patients with none,

coupled with the CAPRA-S score risk groups. Interestingly, the PTEN deletion status identified patients of CAPRA-S low-risk group who have an increased risk of biochemical recurrence, while the $16 \mathrm{p} 13.3$ gain status alone did not further stratify the low-risk group as we reported [18]. These results are in agreement with previous studies showing that combinations of genomic features such as gene expression changes and copy number alterations, including PTEN deletion status, can add prognostic information to the CAPRA-S score $[30,31]$. Owing to the relatively small number of secondary adverse events like metastases and prostate cancer-specific deaths in our cohort, future studies should focus on assessing the clinical significance of this PTEN-16p13.3 co-alteration status with respect to these adverse clinical end-points in large independent cohorts with long clinical follow-up.

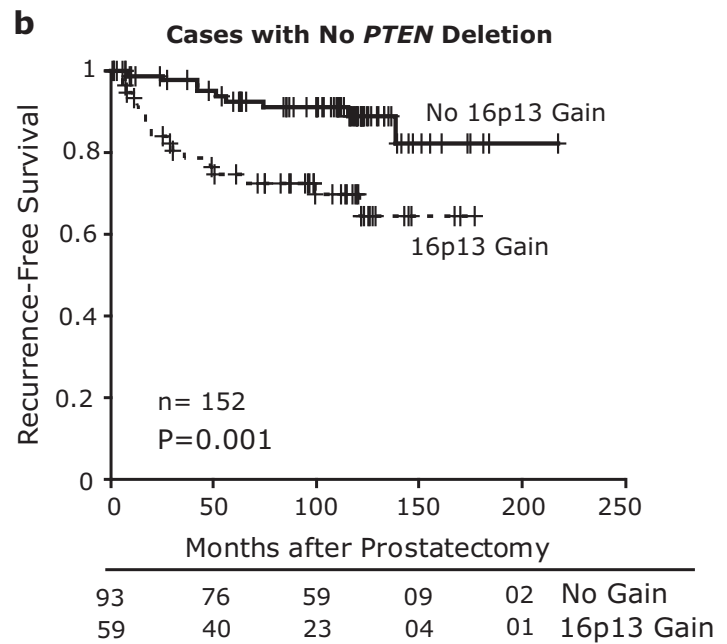

d C-Index of Individual and Combined Markers

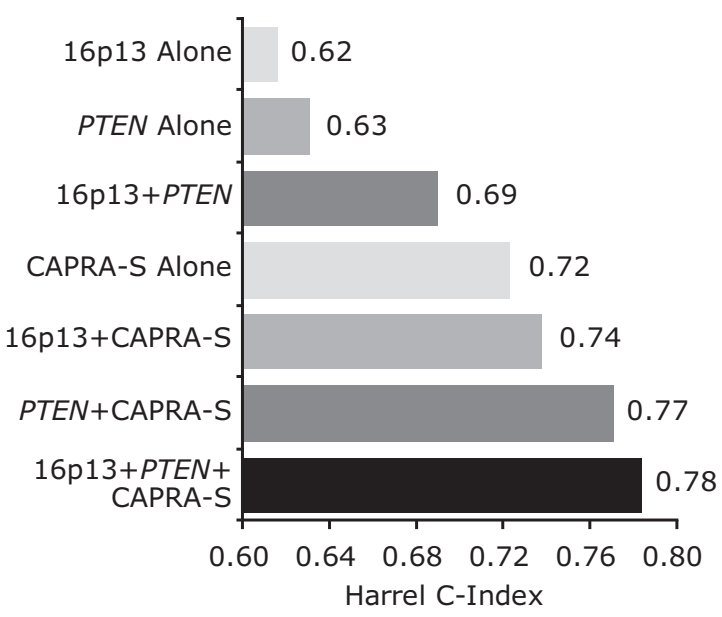

either or both genomic alterations. Censored data (tick marks), number of patients at risk at respective time points, and $P$-value (log-rank test) are indicated. Harrel C-index is improved (d) when combining $16 \mathrm{p} 13$ gain, PTEN deletion and CAPRA-S score risk group, compared to individual markers or either of the three

Given the enhanced risk of biochemical recurrence associated with co-alteration of PTEN and 16p13.3, it is possible that patients with such tumors would benefit of adjuvant treatments. In prostate cancer, the usefulness of adjuvant systemic therapy such as chemotherapy postsurgery remains to be established [32]. The lack of demonstrated effectiveness may be explained in part by differences of tumor biology among patients. Molecular biomarkers such as PTEN/16p13.3 FISH may help patient selection and thus improve the success of future clinical trials. The assessment of these markers retrospectively in samples of patients who have received adjuvant therapy may provide data to support this hypothesis.

PTEN inactivation occurs predominantly via genomic deletion at 10q23 [6,33], which results in increased levels of phosphatidylinositol [3-5]-trisphosphate (PIP3). PIP3 
Table 4 Univariate and multivariate Cox proportional hazard analysis predicting biochemical recurrence for the $P T E N-16 \mathrm{p} 13$ co-alteration status adjusted for standard clinicopathologic parameters (A) and CAPRA-S score (B)

\begin{tabular}{|c|c|c|c|c|}
\hline \multirow[t]{2}{*}{ Variables } & \multicolumn{2}{|l|}{ Univariate analysis } & \multicolumn{2}{|l|}{ Multivariate analysis } \\
\hline & $\begin{array}{l}\text { Hazard ratio ( } 95 \% \\
\text { confidence interval) }\end{array}$ & $P$-value & $\begin{array}{l}\text { Hazard ratio ( } 95 \% \\
\text { confidence interval) }\end{array}$ & $P$-value \\
\hline \multicolumn{5}{|c|}{ (A) Standard clinicopathologic parameters } \\
\hline \multicolumn{5}{|c|}{ PTEN-16p13 co-alteration status } \\
\hline $\begin{array}{l}\text { No PTEN and No } 16 \mathrm{p} 13 \\
\text { (reference) }\end{array}$ & - & $<0.0001$ & - & 0.003 \\
\hline PTEN or $16 \mathrm{p} 13$ & $3.93(1.88-8.25)$ & $<0.0001$ & $2.90(1.35-6.22)$ & 0.006 \\
\hline$P T E N$ and $16 \mathrm{p} 13$ & $6.88(3.14-15.08)$ & $<0.0001$ & $4.18(1.82-9.59)$ & 0.001 \\
\hline $\begin{array}{l}\text { Preoperative prostate- } \\
\text { specific antigen }^{\mathrm{a}}\end{array}$ & $1.08(1.05-1.10)$ & $<0.0001$ & $1.05(1.02-1.08)$ & 0.002 \\
\hline \multicolumn{5}{|l|}{ Gleason grade } \\
\hline $\begin{array}{l}\text { Group 3-5 }(\geq 4+3) \text { vs. } \\
\text { group } 1-2(\leq 3+4)\end{array}$ & $4.70(2.79-7.90)$ & $<0.0001$ & $2.16(1.14-4.12)$ & 0.019 \\
\hline pT-stage (T3 vs. T2) & $3.29(1.98-5.48)$ & $<0.0001$ & $1.46(0.80-2.67)$ & 0.217 \\
\hline $\begin{array}{l}\text { Surgical margin (positive vs. } \\
\text { negative) }\end{array}$ & $2.25(1.37-3.72)$ & 0.002 & $1.50(0.87-2.57)$ & 0.143 \\
\hline Age at surgery ${ }^{\mathrm{a}}$ & $1.03(0.98-1.07)$ & 0.263 & $0.98(0.96-1.05)$ & 0.924 \\
\hline \multicolumn{5}{|l|}{ (B) CAPRA-S score } \\
\hline \multicolumn{5}{|c|}{ PTEN-16p13 co-alteration status } \\
\hline $\begin{array}{l}\text { No PTEN and no } 16 \mathrm{p} 13 \\
\text { (reference) }\end{array}$ & - & $<0.0001$ & - & 0.001 \\
\hline PTEN or $16 \mathrm{p} 13$ & $3.93(1.88-8.25)$ & $<0.0001$ & $3.55(1.69-7.46)$ & 0.001 \\
\hline PTEN and $16 \mathrm{p} 13$ & $6.88(3.14-15.08)$ & $<0.0001$ & $4.70(2.12-10.42)$ & $<0.0001$ \\
\hline \multicolumn{5}{|l|}{ CAPRA-S risk } \\
\hline Low $(0-2)$ reference & - & $<0.0001$ & - & $<0.0001$ \\
\hline Intermediate (3-5) & $3.21(1.66-6.19)$ & 0.001 & $2.70(1.40-5.23)$ & 0.003 \\
\hline $\operatorname{High}(\geq 6)$ & $9.25(4.58-18.69)$ & $<0.0001$ & $7.34(3.57-15.07)$ & $<0.0001$ \\
\hline
\end{tabular}

${ }^{a}$ Analyzed as a continuous variable; $P$-value: Wald test triggers the phosphorylation and activation of AKT by PDK1 leading to the stimulation of pathways related to cell growth and survival [34]. Our laboratory has identified PDPK1 encoding PDK1 at $16 \mathrm{p} 13.3$ as a potential driver of the gain and found that PDK1 stimulates prostate cancer cell migration in vitro [8]. Increasing data from the literature indicate that PDK1 not only phosphorylates AKT, but is also directly involved in cell invasion and migration [35]. It is possible that an overexpression of PDK1 resulting from 16p13.3 gain would potentialize the PIK3/AKT pathway activation and/or provide additional advantages relevant to tumor progression such as an increased cell motility leading to tumor cell spreading beyond the prostate, which may explain the augmented risk of tumor recurrence associated with both PTEN and $16 p 13.3$ copy number alterations. Assessment of the in situ expression and activation status of the proteins involved in the PIK3/AKT pathway as well as further work on animal models are warranted to elucidate the role of PDK1 in PCa and validate this hypothesis.
Genomic instability, as reflected by the percentage of tumor genome harboring copy number alterations, has been shown to be associated with adverse outcome [36, 37]. It is thus possible that the higher risk of biochemical recurrence associated with PTEN-16p13.3 co-alteration reflects an increased genomic instability. PTEN has been shown to contribute to genomic instability leading to aggressive prostate cancer in animal models [38]. In our previous CNA analysis, we identified 8q24 gain (MYC) and 16q23 deletion along PTEN deletion and $16 \mathrm{p} 13$ gain as the four most common alterations enriched in lymph node metastases [7]. It has been shown that the co-deletion of PTEN and 16q23 was associated with poor outcome after radical prostatectomy [39]. It would be interesting to explore whether these other key copy number alterations would provide additional prognostic value, in particular MYC that synergizes with PTEN for tumor initiation and progression in animal models [38].

A potential use for such a panel of copy number alterations would be as FISH biomarkers on diagnostic biopsies 
to identify more effectively patients suitable for active surveillance, ultimately improving the pretreatment prognostication given that accurate Gleason grade on biopsies can be challenging to obtain. PTEN deletion detected in prostate needle biopsies of Gleason score 6 (grade group 1) has been shown to be associated with upgrading to Gleason score 7+ (grade group 2 and up) at radical prostatectomy [40], which suggests that molecular biomarkers may overcome some of the limitations associated with the standard histopathologic evaluation of biopsy specimens. Whether the improved patient risk stratification afforded by combining PTEN/16p13.3 in our study would hold true in biopsy specimens from an active surveillance cohort remains to be demonstrated.

FISH is regarded as the gold standard for the assessment of copy number alterations in tissue specimens owing to its ability to delineate specific genomic events with a spatial resolution facilitating a sensitive evaluation of individual cancer foci at a single-cell level [41, 42]. Assessing the expression of encoded proteins by immunohistochemistry is considered as an alternative approach to capture the prognostic value associated with copy number alterations. Previous PTEN immunohistochemistry assays applied to large cohorts have yield variable results in terms of outcome prediction and correlation with FISH [14, 43]. An improved PTEN immunohistochemistry assay was applied recently to one of the cohorts previously analyzed by immunohistochemistry and FISH mentioned above [14] and the authors reported a sensitivity of $83 \%$ and $67 \%$ to respectively detect homozygous and hemizygous deletion [44]. While PTEN protein loss assessed by immunohistochemistry was associated with increased risk of biochemical recurrence, a further risk stratification was achieved by combining FISH with immunohistochemistry results, substantiating the enhanced value of PTEN FISH in outcome prediction. Future studies comparing both FISH and immunohistochemistry on additional cohorts, including ours, would be important to confirm these observations.

In summary, the results of our study support the prognostic value of PTEN deletion in prostate cancer, which can be further improved in combination with 16 p13.3 gain status, suggesting that these genomic alterations may cooperatively contribute to prostate cancer progression. DNA copy number analysis of PTEN and 16 p13.3 could be of important clinical value particularly for preoperative risk assessment of the clinically most challenging group of low-grade and intermediate-grade prostate cancer.

Acknowledgements We would like to thank Walead Ebrahimizadeh, a member of J. Lapointe's laboratory, for his constructive feedback and discussion during the execution of this project. This work was supported by the US Department of Defense (DoD, W81XWH-11-10638) and the Fonds de Recherche du Québec-Santé (FRQS, Chercheur boursier) to JL and by the Canadian Institutes of Health Research (CIHR) System Biology and McGill Division of Urology studentships to YB.

\section{Compliance with ethical standards}

Conflict of interest The authors declare that they have no conflict of interest.

\section{References}

1. Siegel RL, Miller KD, Jemal A. Cancer Statistics, 2017. CA Cancer J Clin 2017;67:7-30.

2. Punnen S, Cooperberg MR. The epidemiology of high-risk prostate cancer. Current opinion in urology 2013;23:331-6

3. Klotz L, Vesprini D, Sethukavalan P, Jethava V, Zhang L, Jain S, et al. Long-term follow-up of a large active surveillance cohort of patients with prostate cancer. Journal of Clinical Oncology 2014;33:272-7.

4. Chang AJ, Autio KA, Roach M, 3rd, Scher HI. High-risk prostate cancer-classification and therapy. Nat Rev Clin Oncol 2014;11:308-23.

5. Epstein JI, Feng Z, Trock BJ, Pierorazio PM. Upgrading and downgrading of prostate cancer from biopsy to radical prostatectomy: incidence and predictive factors using the modified Gleason grading system and factoring in tertiary grades. European urology 2012;61:1019-24.

6. Cancer Genome Atlas Research Network. The Molecular Taxonomy of Primary Prostate Cancer. Cell 2015;163:1011-25.

7. Lapointe J, Li C, Giacomini CP, Salari K, Huang S, Wang P, et al. Genomic profiling reveals alternative genetic pathways of prostate tumorigenesis. Cancer Res 2007;67:8504-10.

8. Choucair KA, Guerard KP, Ejdelman J, Chevalier S, Yoshimoto M, Scarlata E, et al. The 16p13.3 (PDPK1) Genomic Gain in Prostate Cancer: A Potential Role in Disease Progression. Translational oncology 2012;5:453-60.

9. Li J, Yen C, Liaw D, Podsypanina K, Bose S, Wang SI, et al. PTEN, a putative protein tyrosine phosphatase gene mutated in human brain, breast, and prostate cancer. Science 1997;275:19437.

10. Myers MP, Stolarov JP, Eng C, Li J, Wang SI, Wigler MH, et al. P-TEN, the tumor suppressor from human chromosome 10q23, is a dual-specificity phosphatase. Proc Natl Acad Sci U S A 1997;94:9052-7.

11. Stambolic V, Suzuki A, de la Pompa JL, Brothers GM, Mirtsos C, Sasaki T, et al. Negative regulation of PKB/Akt-dependent cell survival by the tumor suppressor PTEN. Cell 1998;95:29-39.

12. Alessi DR, Deak M, Casamayor A, Caudwell FB, Morrice N, Norman DG, et al. 3-Phosphoinositide-dependent protein kinase-1 (PDK1): structural and functional homology with the Drosophila DSTPK61 kinase. Curr Biol 1997;7:776-89.

13. Choucair K, Ejdelman J, Brimo F, Aprikian A, Chevalier S, Lapointe J. PTEN genomic deletion predicts prostate cancer recurrence and is associated with low AR expression and transcriptional activity. BMC Cancer 2012;12:543.

14. Krohn A, Diedler T, Burkhardt L, Mayer PS, De Silva C, MeyerKornblum M, et al. Genomic deletion of PTEN is associated with tumor progression and early PSA recurrence in ERG fusionpositive and fusion-negative prostate cancer. Am J Pathol 2012;181:401-12. 
15. Qu X, Jeldres C, Glaskova L, Friedman C, Schroeder S, Nelson PS, et al. Identification of Combinatorial Genomic Abnormalities Associated with Prostate Cancer Early Recurrence. The Journal of molecular diagnostics : JMD 2016;18:215-24.

16. Troyer DA, Jamaspishvili T, Wei W, Feng Z, Good J, Hawley S, et al. A multicenter study shows PTEN deletion is strongly associated with seminal vesicle involvement and extracapsular extension in localized prostate cancer. Prostate 2015;75:206-15.

17. Yoshimoto M, Cunha IW, Coudry RA, Fonseca FP, Torres CH, Soares FA, et al. FISH analysis of 107 prostate cancers shows that PTEN genomic deletion is associated with poor clinical outcome. British journal of cancer 2007;97:678-85.

18. Bramhecha YM, Guerard KP, Rouzbeh S, Scarlata E, Brimo F, Chevalier S, et al. Genomic Gain of 16p13.3 in Prostate Cancer Predicts Poor Clinical Outcome after Surgical Intervention. Mol Cancer Res 2018;16:115-23.

19. McShane LM, Altman DG, Sauerbrei W, Taube SE, Gion M, Clark GM. REporting recommendations for tumor MARKer prognostic studies (REMARK). Nature clinical practice Urology 2005;2:416-22.

20. Humphrey PA, Moch H, Cubilla AL, Ulbright TM, Reuter VE. The 2016 WHO Classification of Tumours of the Urinary System and Male Genital Organs-Part B: Prostate and Bladder Tumours. European urology 2016;70:106-19.

21. Cooperberg MR, Hilton JF, Carroll PR. The CAPRA-S score: A straightforward tool for improved prediction of outcomes after radical prostatectomy. Cancer 2011;117:5039-46.

22. Punnen S, Freedland SJ, Presti JC, Jr., Aronson WJ, Terris MK, Kane CJ, et al. Multi-institutional validation of the CAPRA-S score to predict disease recurrence and mortality after radical prostatectomy. European urology 2014;65:1171-7.

23. Harrell FE, Jr., Lee KL, Mark DB. Multivariable prognostic models: issues in developing models, evaluating assumptions and adequacy, and measuring and reducing errors. Stat Med 1996;15:361-87.

24. Bismar TA, Yoshimoto M, Vollmer RT, Duan Q, Firszt M, Corcos J, et al. PTEN genomic deletion is an early event associated with ERG gene rearrangements in prostate cancer. BJU international 2011;107:477-85.

25. Han B, Mehra R, Lonigro RJ, Wang L, Suleman K, Menon A, et al. Fluorescence in situ hybridization study shows association of PTEN deletion with ERG rearrangement during prostate cancer progression. Mod Pathol 2009;22:1083-93.

26. Kwabi-Addo B, Giri D, Schmidt K, Podsypanina K, Parsons R, Greenberg N, et al. Haploinsufficiency of the Pten tumor suppressor gene promotes prostate cancer progression. Proc Natl Acad Sci U S A 2001;98:11563-8.

27. Whang YE, Wu X, Suzuki H, Reiter RE, Tran C, Vessella RL, et al. Inactivation of the tumor suppressor PTEN/MMAC1 in advanced human prostate cancer through loss of expression. Proc Natl Acad Sci U S A 1998;95:5246-50.

28. Qu X, Randhawa G, Friedman C, Kurland BF, Glaskova L, Coleman I, et al. A three-marker FISH panel detects more genetic aberrations of AR, PTEN and TMPRSS2/ERG in castrationresistant or metastatic prostate cancers than in primary prostate tumors. PLoS One 2013;8:e74671.

29. Lotan TL, Gurel B, Sutcliffe S, Esopi D, Liu W, Xu J, et al. PTEN protein loss by immunostaining: analytic validation and prognostic indicator for a high risk surgical cohort of prostate cancer patients. Clin Cancer Res 2011;17:6563-73.
30. Cooperberg MR, Davicioni E, Crisan A, Jenkins RB, Ghadessi M, Karnes RJ. Combined value of validated clinical and genomic risk stratification tools for predicting prostate cancer mortality in a high-risk prostatectomy cohort. European urology 2015;67:32633.

31. Lennartz M, Minner S, Brasch S, Wittmann H, Paterna L, Angermeier K, et al. The Combination of DNA Ploidy Status and PTEN/6q15 Deletions Provides Strong and Independent Prognostic Information in Prostate Cancer. Clin Cancer Res 2016;22:2802-11.

32. Pignot G, Maillet D, Gross E, Barthelemy P, Beauval JB, Constans-Schlurmann F, et al. Systemic treatments for high-risk localized prostate cancer. Nat Rev Urol 2018.

33. Cairns P, Okami K, Halachmi S, Halachmi N, Esteller M, Herman $\mathrm{JG}$, et al. Frequent inactivation of PTEN/MMAC1 in primary prostate cancer. Cancer Res 1997;57:4997-5000.

34. Toren P, Zoubeidi A. Targeting the PI3K/Akt pathway in prostate cancer: challenges and opportunities (review). Int $\mathrm{J}$ Oncol 2014;45:1793-801.

35. Gagliardi PA, di Blasio L, Primo L. PDK1: A signaling hub for cell migration and tumor invasion. Biochimica et biophysica acta 2015;1856:178-88.

36. Hieronymus H, Schultz N, Gopalan A, Carver BS, Chang MT, Xiao Y, et al. Copy number alteration burden predicts prostate cancer relapse. Proc Natl Acad Sci U S A 2014;111: 11139-44.

37. Lalonde E, Ishkanian AS, Sykes J, Fraser M, Ross-Adams H, Erho N, et al. Tumour genomic and microenvironmental heterogeneity for integrated prediction of 5-year biochemical recurrence of prostate cancer: a retrospective cohort study. The Lancet 2014;15:1521-32.

38. Hubbard GK, Mutton LN, Khalili M, McMullin RP, Hicks JL, Bianchi-Frias D, et al. Combined MYC Activation and Pten Loss Are Sufficient to Create Genomic Instability and Lethal Metastatic Prostate Cancer. Cancer Res 2016;76:283-92.

39. Kluth M, Runte F, Barow P, Omari J, Abdelaziz ZM, Paustian L, et al. Concurrent deletion of 16q23 and PTEN is an independent prognostic feature in prostate cancer. Int J Cancer 2015;137:235463.

40. Picanco-Albuquerque CG, Morais CL, Carvalho FL, Peskoe SB, Hicks JL, Ludkovski O, et al. In prostate cancer needle biopsies, detections of PTEN loss by fluorescence in situ hybridization (FISH) and by immunohistochemistry (IHC) are concordant and show consistent association with upgrading. Virchows Arch 2016;468:607-17.

41. Bishop R. Applications of fluorescence in situ hybridization (FISH) in detecting genetic aberrations of medical significance. Bioscience Horizons: The International Journal of Student Research 2010;3:85-95.

42. Gozzetti A, Le Beau MM. Fluorescence in situ hybridization: uses and limitations. Seminars in hematology 2000;37:320-33.

43. Cuzick J, Yang ZH, Fisher G, Tikishvili E, Stone S, Lanchbury JS, et al. Prognostic value of PTEN loss in men with conservatively managed localised prostate cancer. British journal of cancer 2013;108:2582-9.

44. Lotan TL, Heumann A, Rico SD, Hicks J, Lecksell K, Koop C, et al. PTEN loss detection in prostate cancer: comparison of PTEN immunohistochemistry and PTEN FISH in a large retrospective prostatectomy cohort. Oncotarget 2017;8:6556676. 